\title{
Anisotropy of Superconducting Single Crystal SmFeAsO0.8F0.2 Studied by Torque Magnetometry
}

\section{Journal Article}

\section{Author(s):}

Weyeneth, S.; Puzniak, R.; Mosele, U.; Zhigadlo, N. D.; Katrych, S.; Bukowski, Z.; Karpinski, J.; Kohout, S.; Roos, J.; Keller, H.

Publication date:

2009

Permanent link:

https://doi.org/10.3929/ethz-b-000017819

\section{Rights / license:}

In Copyright - Non-Commercial Use Permitted

\section{Originally published in:}

Journal of Superconductivity and Novel Magnetism 22(4), https://doi.org/10.1007/s10948-008-0413-1 


\title{
Anisotropy of Superconducting Single Crystal $\mathrm{SmFeAsO}_{0.8} \mathrm{~F}_{0.2}$ Studied by Torque Magnetometry
}

\author{
S. Weyeneth · R. Puzniak • U. Mosele • N.D. Zhigadlo • \\ S. Katrych · Z. Bukowski • J. Karpinski • S. Kohout • \\ J. Roos $\cdot$ H. Keller
}

Received: 21 November 2008 / Accepted: 28 November 2008 / Published online: 24 December 2008

(C) Springer Science+Business Media, LLC 2008

\begin{abstract}
Single crystals of the oxypnictide superconductor $\mathrm{SmFeAsO}_{0.8} \mathrm{~F}_{0.2}$ with $T_{\mathrm{c}} \simeq 45(1) \mathrm{K}$ were investigated by torque magnetometry. The crystals of mass $\leq 0.1 \mu \mathrm{g}$ were grown by a high-pressure cubic anvil technique. The use of a high-sensitive piezoresistive torque sensor made it possible to study the anisotropic magnetic properties of these tiny crystals. The anisotropy parameter $\gamma$ was found to be field independent, but varies strongly with temperature ranging from $\gamma \simeq 8$ at $T \lesssim T_{\mathrm{c}}$ to $\gamma \simeq 23$ at $T \simeq 0.4 T_{\mathrm{c}}$. This unusual behavior of $\gamma$ signals unconventional superconductivity.
\end{abstract}

Keywords Oxypnictides · Anisotropy · Multi-band superconductivity $\cdot$ Single crystal $\cdot$ Torque magnetometry

PACS 74.70.Dd $\cdot$ 74.25.Ha $\cdot$ 74.20.De

\section{Introduction}

The search for novel superconductors recently led to the discovery of the Fe-based oxypnictide layered superconductor $\mathrm{LaFeAsO}_{1-x} \mathrm{~F}_{x}$ with a transition temperature of $T_{\mathrm{c}} \simeq 26 \mathrm{~K}$ [1]. By substituting La with other rare earth ions, like Sm,

S. Weyeneth $(\varangle) \cdot$ U. Mosele $\cdot$ S. Kohout $\cdot$ J. Roos $\cdot$ H. Keller Physik-Institut der Universität Zürich, Winterthurerstrasse 190, 8057 Zürich, Switzerland

e-mail:wstephen@physik.uzh.ch

R. Puzniak

Institute of Physics, Polish Academy of Sciences, Aleja Lotników

32/46, 02-668 Warsaw, Poland

N.D. Zhigadlo $\cdot$ S. Katrych · Z. Bukowski · J. Karpinski Laboratory for Solid State Physics, ETH Zürich, 8093 Zürich, Switzerland
$\mathrm{Ce}, \mathrm{Nd}, \mathrm{Pr}$, or $\mathrm{Gd}$, a series of novel superconducting materials were synthesized with $T_{\mathrm{c}}$ up to $55 \mathrm{~K}$ [2-7]. These compounds have a layered crystal structure consisting of $\mathrm{LaO}$ and FeAs sheets, where the $\mathrm{Fe}$ ions are arranged on simple square lattices [1]. According to theoretical predictions, superconductivity takes place in the FeAs layers, whereas the $\mathrm{LaO}$ layers are charge reservoirs when doped with $\mathrm{F}$ ions $[8,9]$.

The anisotropic behavior of layered superconductors can be characterized by their effective mass anisotropy $\gamma$, which in the framework of the classical anisotropic GinzburgLandau theory is given by $\gamma=\sqrt{m_{c}^{*} / m_{a b}^{*}}=\lambda_{c} / \lambda_{a b}=$ $B_{c 2}^{a b} / B_{c 2}^{c}$ [10]. Here $m_{a b}^{*}$ and $m_{c}^{*}$ are the effective carrier masses related to supercurrents flowing in the $a b$-planes and along the $c$-axis, respectively; $\lambda_{a b}$ and $\lambda_{c}$ are the corresponding magnetic penetration depths; and $B_{c 2}^{a b}$ and $B_{c 2}^{c}$ the corresponding upper critical fields. In order to determine reliable values of $\gamma$, high-quality single crystals are required.

An estimation of $\gamma \geq 30$ at $T=0 \mathrm{~K}$ was made for $\mathrm{SmFeAsO}_{0.82} \mathrm{~F}_{0.18}$ from measurements of the $c$-axis plasma frequency using infrared ellipsometry [11]. From pointcontact spectroscopy [12] and resistivity experiments [13] on $\mathrm{LaFeAsO}_{0.9} \mathrm{~F}_{0.1-\delta}$ the anisotropy parameter was estimated to be $\gamma \simeq 10$. Recent resistivity measurements on single crystal $\mathrm{NdFeAsO}_{0.82} \mathrm{~F}_{0.18}$ revealed a value of $\gamma \lesssim 5$ [14] close to $T_{\mathrm{c}}$. Band structure calculations for $\mathrm{LaFeAsO}_{1-x} \mathrm{~F}_{x}$ yield a resistivity anisotropy of $\sim 15$ for isotropic scattering, corresponding to $\gamma \simeq 4$ [8]. For comparison, in $\mathrm{MgB}_{2}$, the value of $\gamma$ ranges from 1 to 8 and shows a strong temperature and field dependence $[15,16]$. This unconventional behavior of $\gamma$ is well described within the framework of twoband superconductivity $[17,18]$. 


\section{Experiment}

Here we report magnetic torque experiments on a single crystal of $\mathrm{SmFeAsO}_{0.8} \mathrm{~F}_{0.2}$ with a $T_{\mathrm{c}} \simeq 45(1) \mathrm{K}$ performed in the temperature range of 19 to $45 \mathrm{~K}$ and in magnetic fields up to $1.4 \mathrm{~T}$. From these measurements the magnetic anisotropy $\gamma$ was extracted in the framework of the anisotropic London model [19, 20]. Single crystals of nominal composition $\mathrm{SmFeAsO}_{0.8} \mathrm{~F}_{0.2}$ [21] with masses of only $\approx 0.1 \mu \mathrm{g}$ were grown from $\mathrm{NaCl} / \mathrm{KCl}$ flux using a highpressure cubic anvil technique [22, 23]. The plate-like crystals used in this work are of rectangular shape with typical dimensions of $70 \times 25 \times 3 \mu^{3}$, corresponding to an estimated volume of $V \simeq 5 \times 10^{-15} \mathrm{~m}^{3}$. The crystal structure was checked by means of $\mathrm{X}$-ray diffraction revealing the $c$ axis to be perpendicular to the plates.

Selected crystals of $\mathrm{SmFeAsO}_{0.8} \mathrm{~F}_{0.2}$ of the same batch with $T_{\mathrm{c}} \simeq 45 \mathrm{~K}$ were characterized in a commercial Quantum Design Magnetometer MPMS XL. As an example, Fig. 1 shows the zero-field cooled (ZFC) magnetic moment $m$ of one of the crystals vs. temperature in $1 \mathrm{mT}$ parallel to the $c$-axis. The rough estimate for $T_{\mathrm{c}} \simeq 45(1) \mathrm{K}$ (straight line in Fig. 1) is consistent with $T_{\mathrm{c}}=46.0$ (3) $\mathrm{K}$ obtained from the temperature dependence of the in-plane magnetic penetration depth $\lambda_{a b}$ (see later Fig. 4). The magnetic moment $m$ saturates in the Meissner state below $40 \mathrm{~K}$ and the total transition width is $\Delta T \lesssim 4 \mathrm{~K}$, demonstrating the good quality of the sample.

The magnetic torque $\tau$ of a single-crystal sample in a static magnetic field $B$ applied under a certain angle $\theta$ with respect to the crystallographic $c$-axis of the crystal is given by $\vec{\tau}=\vec{m} \times \vec{B}=\mu_{0}(\vec{m} \times \vec{H})$, or equivalently $\tau(\theta)=$ $m(\theta) B \sin (\phi(\theta))$. Here $\vec{m}$ denotes the magnetic moment of the sample and $\phi(\theta)$ the respective angle between $\vec{B}$ and $\vec{m}$.

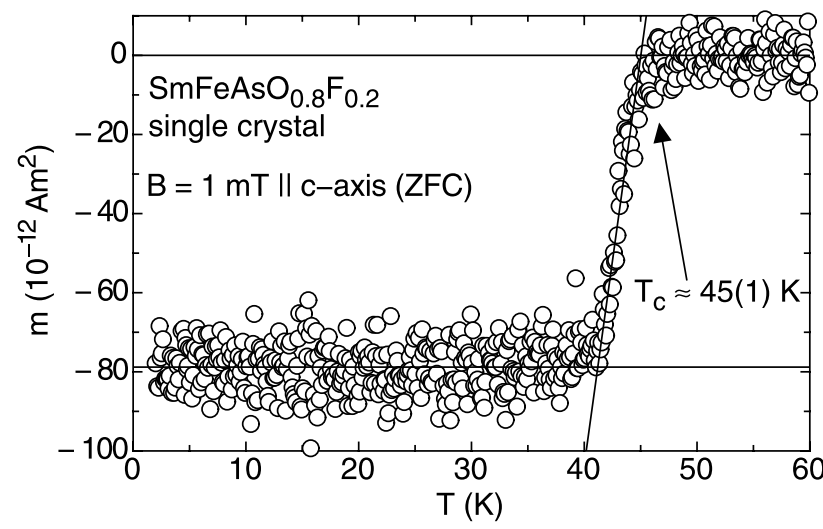

Fig. 1 Temperature dependence of the magnetic moment $m$ of a $\mathrm{SmFeAsO}_{0.8} \mathrm{~F}_{0.2}$ single crystal measured in $1 \mathrm{mT}$ parallel to the $c$-axis (zero-field cooling, ZFC). The rough estimate of the transition temperature $T_{\mathrm{c}} \simeq 45$ (1) $\mathrm{K}$ (indicated by the arrow) is consistent with the value of $T_{\mathrm{c}}=46.0$ (3) $\mathrm{K}$ determined from the temperature dependence of the in-plane magnetic penetration depth $\lambda_{a b}$ (see text and Fig. 4)
Our apparatus was especially designed for measurements on micro-crystals with masses smaller than $1 \mu \mathrm{g}$ using micromachined sensors of high sensitivity $\left(\sim 10^{-14} \mathrm{~N} \mathrm{~m}\right)$ developed in our group [24]. To collect angular dependent torque data $\tau(\theta)$, we used a measurement system with a turnable Bruker NMR iron yoke magnet with a maximum magnetic field of $1.4 \mathrm{~T}$, allowing a rotation of the magnetic field direction of more that $360^{\circ}$ with respect to a crystallographic axis of the sample. Temperatures down to $10 \mathrm{~K}$ can be achieved with a flow cryostat between the poles of the magnet. The possibility of rotating the magnetic field around a fixed sample increases the sensitivity again because background effects to the torque are minimized.

Below $T_{\mathrm{c}}$ we observe a torque signal arising from the interaction of the applied magnetic field with vortices in the sample. Based on the 3D anisotropic London model, the free energy of an anisotropic superconductor in the mixed state was calculated by Kogan et al. [19, 20]:

$\tau(\theta)=-\frac{V \Phi_{0} B}{16 \pi \mu_{0} \lambda_{a b}^{2}}\left(1-\frac{1}{\gamma^{2}}\right) \frac{\sin (2 \theta)}{\epsilon(\theta)} \ln \left(\frac{\eta B_{c 2}^{c}}{\epsilon(\theta) B}\right)$.

$V$ is the volume of the crystal, $\Phi_{0}$ is the elementary flux quantum, $B_{c 2}^{c}$ is the upper critical field along the $c$-axis of the crystal, $\eta$ denotes a numerical parameter of the order unity depending on the structure of the flux-line lattice, and $\epsilon(\theta)=\left[\cos ^{2}(\theta)+\gamma^{-2} \sin ^{2}(\theta)\right]^{1 / 2}$. By measuring the angular dependence of the torque in the mixed state of a superconductor, three fundamental parameters can be extracted from the data: the in-plane magnetic penetration depth $\lambda_{a b}$, the $c$-axis upper critical field $B_{c 2}^{c}$, and the effective mass anisotropy $\gamma$. In this work we performed angledependent measurements of the torque over more than $180^{\circ}$ in order to investigate the full angular dependent magnetization in terms of (1). The torque was measured with a clockwise and a counterclockwise rotating magnetic field and then averaged according to $\tau_{\text {rev }}=\left(\tau\left(\theta^{+}\right)+\tau\left(\theta^{-}\right)\right) / 2$ to reduce vortex pinning effects (see upper panel of Fig. 2). To further minimize the influence of pinning, we partly applied a vortex-lattice shaking technique [25]. With this technique the relaxation of the vortex-lattice towards its thermal equilibrium is strongly enhanced by using an additional small magnetic AC field perpendicular to the static external field $B$. The magnitude of the AC field was $\simeq 5 \mathrm{mT}$ and the shaking frequency was $\simeq 200 \mathrm{~Hz}$. With these parameters the best experimental conditions were obtained, including the vortex-lattice relaxation towards thermal equilibrium. Examples of the reversible torque as a function of the angle $\theta$ determined in the superconducting state are displayed in the lower panel of Fig. 2. A small temperature independent background of $10^{-12} \mathrm{~N}$ m was recorded well above $T_{\mathrm{c}}$ and subtracted from all measurements below $T_{\mathrm{c}}$.

The most reliable parameter which can be extracted from the torque data is the anisotropy parameter $\gamma$ because, according to (1), it only depends on the angular dependence 

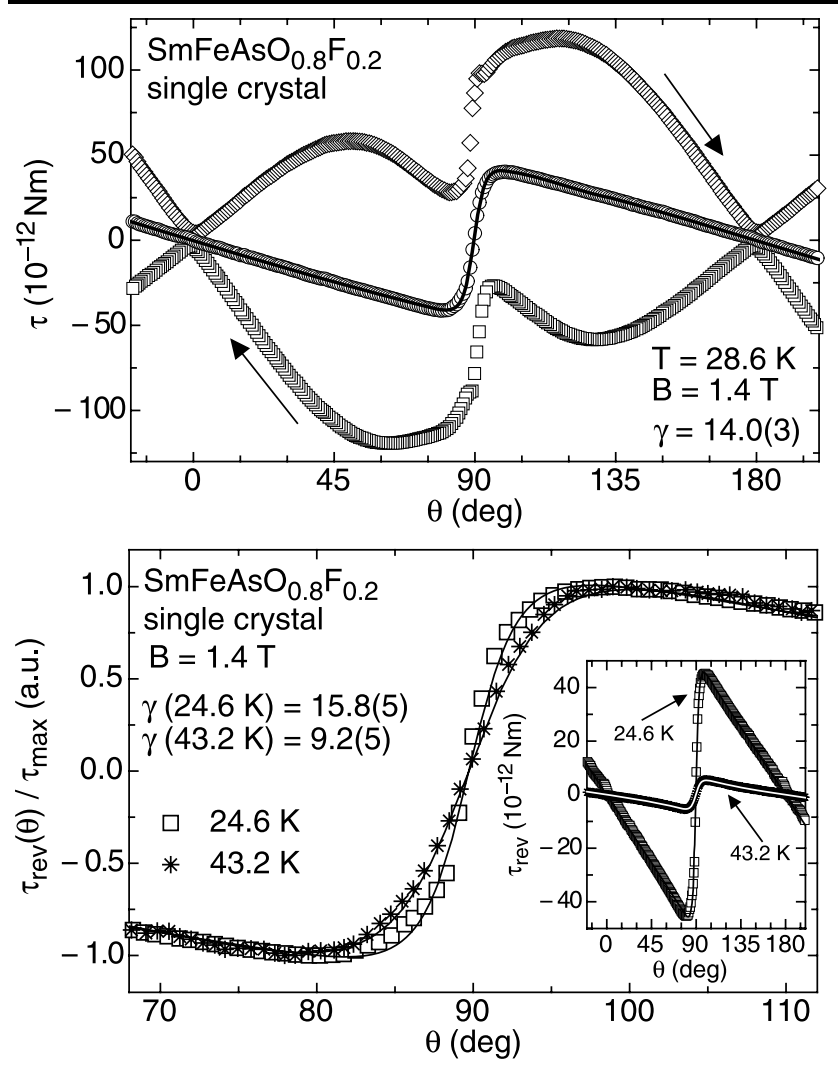

Fig. 2 Upper panel: Angular dependence of the reversible torque of a single crystal of $\mathrm{SmFeAsO}_{0.8} \mathrm{~F}_{0.2}$ at $T=28.6 \mathrm{~K}$ and $B=1.4 \mathrm{~T}$. The squares (diamonds) denote a counterclockwise (clockwise) rotating of the magnetic field around the sample. For minimizing pinning effects the mean torque $\tau_{\mathrm{rev}}=\left(\tau\left(\theta^{+}\right)+\tau\left(\theta^{-}\right)\right) / 2$ was calculated (circles). The solid line denotes a fit to (1) from which the anisotropy parameter $\gamma=14.0(3)$ was extracted. Lower panel: Normalized angular dependence of the torque for $\theta$ in the neighborhood of $90^{\circ}$ ( $B$ parallel $a b$-plane) at $24.6 \mathrm{~K}$ (squares) and $43.2 \mathrm{~K}$ (stars). The solid lines represent fits to (1). The different shapes of the two torque signals reflect the temperature dependence of $\gamma$. The inset displays the full angular dependence of the same torque signals

of the torque and not on the sample volume. Figure 2 (lower panel) displays two examples of torque measurements performed at $B=1.4 \mathrm{~T}$ and for two different temperatures, 24.6 and $43.2 \mathrm{~K}$, yielding quite different values of $\gamma$, namely $\gamma(24.6 \mathrm{~K})=15.8(5)$ and $\gamma(43.2 \mathrm{~K})=9.2(5)$. This is clearly reflected in the different shapes of the torque signals for $\theta$ in the neighborhood of $90^{\circ}$ ( $B$ almost parallel to the $a b$-plane). The temperature dependence of $\gamma$ determined at $1.4 \mathrm{~T}$ is displayed in Fig. 3. As the temperature decreases, $\gamma$ rises from $\gamma(45.0 \mathrm{~K})=8.0(7)$ to $\gamma(19.0 \mathrm{~K})=$ 22.6(15). Note that the present values of $\gamma$ are within the range of the values so far reported in the literature $[8,11-$ 14]. At low temperatures, pinning effects give rise to the rather large errors in the anisotropy parameter, preventing a reliable determination of $\gamma$ for $T<19 \mathrm{~K}$. In order to demonstrate that the strong temperature dependence of $\gamma$ is not an artefact of the increasing pinning strength at low tem-

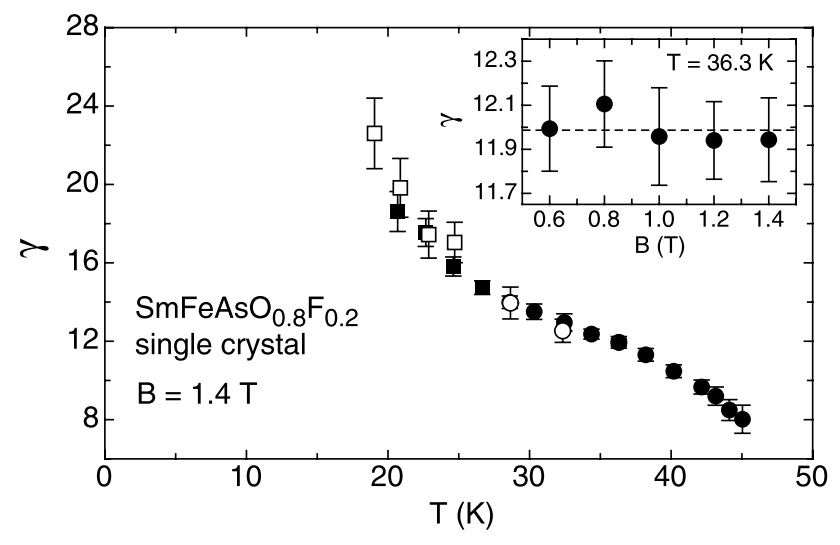

Fig. 3 Temperature dependence of the anisotropy parameter $\gamma$ of a single crystal of $\mathrm{SmFeAsO}_{0.8} \mathrm{~F}_{0.2}$ at $B=1.4 \mathrm{~T}$ obtained from a subsequent analysis of the torque data by means of (1). Full symbols denote values of $\gamma$ extracted from simply averaged data sets, whereas open symbols show values of $\gamma$ from shaked data sets as explained in the text. The values of $\gamma$ obtained from fits to (1) with all parameters free being indicated by circles, whereas the squares represent the values of $\gamma$ evaluated using extrapolated values of $B_{c 2}^{c}$. The inset shows $\gamma$ determined at $36.3 \mathrm{~K}$ from unshaked data at different fields. As can be seen, no field dependence of $\gamma$ is observed

peratures, we compared shaked and unshaked torque data. One would expect that the shaked data are close to the reversible thermal equilibrium state. However, as indicated by the open and closed symbols in Fig. 3, there is within error bars no difference between the values of $\gamma$ determined with and without shaking, attesting that the used procedure to determine $\gamma$ below the irreversibility line is still reliable [25]. Furthermore, the field dependence of $\gamma$ was studied at $T=36.3 \mathrm{~K}$. As shown in the inset of Fig. 3, for $0.6 \mathrm{~T} \leq B \leq 1.4 \mathrm{~T}$ no field dependence of $\gamma$ could be detected, suggesting that the measured anisotropy parameter in this work is $\gamma=\lambda_{c} / \lambda_{a b}=B_{c 2}^{a b} / B_{c 2}^{c}$. Since $\gamma$ is fieldindependent, its determination is not appreciably affected by weak intrinsic pinning present in the sample [26, 27].

The only parameter which is difficult to extract from (1) in the presence of weak pinning is the upper critical field $B_{c 2}^{c}$ as it enters only logarithmically into the formula. For this reason we fitted $B_{c 2}^{c}(T)$ above $28 \mathrm{~K}$ according to $\eta B_{c 2}^{c}(T) \approx$ $88 \mathrm{~T}-(1.9 \mathrm{~T} / \mathrm{K}) \cdot T$ and extrapolated values of $B_{c 2}^{c}(T)$ down to $19 \mathrm{~K}$ in order to follow the temperature dependence of $\gamma$ further down in temperature (note that the fitted slope of $d B_{c 2}^{c} / d T \approx-2 \mathrm{~T} / \mathrm{K}$ agrees well with the slope close to $T_{\mathrm{c}}$ reported in [28]). The values obtained from the extrapolated values of $B_{c 2}^{c}$ are displayed in Fig. 3 as squares, whereas the values evaluated by the free fits are represented by circles.

The temperature dependencies of the upper critical field $B_{c 2}^{c}$ and the in-plane magnetic penetration depth $\lambda_{a b}$ as extracted from the torque data, are displayed in Fig. 4. $B_{c 2}^{c}$ follows a linear temperature dependence down to $28 \mathrm{~K}$. The dashed line represents a linear fit in order to extrapolate $B_{c 2}^{c}$ down to $19 \mathrm{~K}$. Using the WHH approximation [29], 


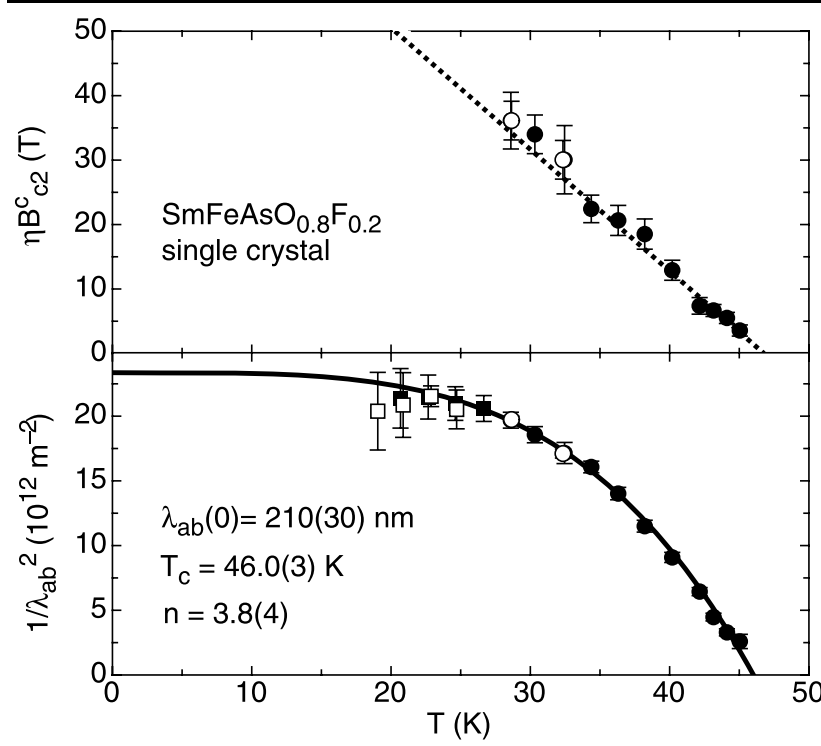

Fig. 4 Upper panel: Temperature dependence of the upper critical field $B_{c 2}^{c}$ of a single crystal of $\mathrm{SmFeAsO}_{0.8} \mathrm{~F}_{0.2}$. The dashed line is a linear fit $\eta B_{c 2}^{c}=88 \mathrm{~T}-(1.9 \mathrm{~T} / \mathrm{K}) \cdot T$. The meaning of the symbols is the same as in Fig. 3. Lower panel: In-plane magnetic penetration depth $\lambda_{a b}(T)$ obtained from the torque data and plotted as $1 / \lambda_{a b}^{2}$ versus temperature. The solid line represents a fit of the data to the power law $1 / \lambda_{a b}^{2}(T)=1 / \lambda_{a b}^{2}(0) \cdot 1-\left(T / T_{\mathrm{c}}^{n}\right)$ with all parameters free. The exponent of $n=3.8$ (4) is close to $n=4$ characteristic for the two-fluid model. The meaning of the symbols is the same as in Fig. 3

$B_{c 2}^{c}(0) \simeq 60 \mathrm{~T}$. The value of $\lambda_{a b}$ was also estimated from the torque data using (1). In the lower panel of Fig. 4 the temperature dependence of $1 / \lambda_{a b}^{2}$ is displayed. As shown by the solid curve in Fig. 4 (lower panel), the data were analyzed by the power law $1 / \lambda_{a b}^{2}(T)=1 / \lambda_{a b}^{2}(0) \cdot\left(1-\left(T / T_{\mathrm{c}}\right)^{n}\right)$ with the free parameters $\lambda_{a b}(0) \simeq 210(30) \mathrm{nm}, T_{\mathrm{c}}=46.0(3) \mathrm{K}$, and $n=3.8(4)$. This value of $\lambda_{a b}(0)$ is in reasonable agreement with the values reported for polycrystalline samples: From $\mu \mathrm{SR}$ experiments Luetkens et al. extracted $\lambda_{a b}(0)=$ 254(2) $\mathrm{nm}$ for $\mathrm{LaFeAsO}_{0.9} \mathrm{~F}_{0.1}$ and $\lambda_{a b}(0)=364(8) \mathrm{nm}$ for $\mathrm{LaFeAsO}_{0.925} \mathrm{~F}_{0.075}$ [30], whereas Khasanov et al. obtained a value of $\lambda_{a b}(0)=198(5) \mathrm{nm}$ for $\mathrm{SmFeAsO}_{0.85}$ [31]. The exponent $n=3.8(4)$ is close to $n=4$ for the two-fluid model, characteristic for a superconductor in the strong-coupling limit [32]. However, the present data are too limited to draw definite conclusions.

$\mathrm{SmFeAsO}_{0.8} \mathrm{~F}_{0.2}$ exhibits a similar temperature dependence of the anisotropy parameter $\gamma$ as $\mathrm{MgB}_{2}$ for which $\gamma$ varies between 1 and 8 , depending on temperature and magnetic field $[15,16,18]$. Therefore, the unusual temperature dependence of $\gamma$ on $\mathrm{SmFeAsO}_{0.8} \mathrm{~F}_{0.2}$, which cannot be explained by conventional Ginzburg-Landau theory, indicates a possible two-gap scenario [18]. Note that other experiments, such as recent resistivity measurements of the upper critical field on $\mathrm{LaFeAsO}_{0.89} \mathrm{~F}_{0.11}$ [28] and NMR studies of $\mathrm{PrFeAsO}_{0.89} \mathrm{~F}_{0.11}$ [33], also suggest that the novel class of Fe-based oxypnictide superconductors exhibit two-band superconductivity. Nevertheless, other possibilities of a temperature dependent $\gamma$ should be mentioned here [26, 27, 34].

\section{Conclusions}

In conclusion, the magnetic anisotropy parameter $\gamma$, and the in-plane magnetic penetration depth $\lambda_{a b}$ were determined by high-sensitive magnetic torque experiments for a single crystal of $\mathrm{SmFeAsO}_{0.8} \mathrm{~F}_{0.2}$. The parameter $\gamma$ extracted from the reversible torque signals was found to be strongly temperature-dependent, ranging from $\gamma \simeq 8$ at $T \lesssim T_{\mathrm{c}}$ to $\gamma \simeq 23$ at $T \simeq 0.4 T_{\mathrm{c}}$. However, no field dependence of $\gamma$ could be detected. For the in-plane magnetic penetration depth at zero temperature a value of $\lambda_{a b}(0) \simeq 210(30) \mathrm{nm}$ was estimated, in agreement with values reported in the literature [30, 31]. The unusual temperature dependence of $\gamma$ suggests that $\mathrm{SmFeAsO}_{0.8} \mathrm{~F}_{0.2}$ is probably a multi-band superconductor, similar to $\mathrm{MgB}_{2}$ [18] and the cuprate superconductors [35]. However, other explanations for this unusual behavior such as e.g. the possibility of an anisotropic $s$-wave gap [34], cannot be ruled out. More detailed experimental work is required to clarify this point.

Acknowledgements The authors are grateful to A. BussmannHolder for very helpful discussions, and to S. Strässle and C. Duttwyler for the help to prepare the manuscript. This work was supported by the Swiss National Science Foundation and in part by the NCCR program MaNEP and the EU Project CoMePhS.

\section{References}

1. Kamihara, Y., Watanabe, T., Hirano, M., Hosono, H.: J. Am. Chem. Soc. 130, 3296 (2008)

2. Chen, X.H., et al.: Nature 453, 761 (2008)

3. Chen, G.F., et al.: Phys. Rev. Lett. 100, 247002 (2008)

4. Ren, Z.-A., et al.: Europhys. Lett. 82, 57002 (2008)

5. Ren, Z.-A., et al.: Mat. Res. Inn. 12, 106 (2008)

6. Cheng, P., et al.: Sci. China G 51, 719 (2008)

7. Ren, Z.-A., et al.: Chin. Phys. Lett. 25, 2215 (2008)

8. Singh, D.J., Du, M.-H.: Phys. Rev. Lett. 100, 237003 (2008)

9. Klauss, H.-H., et al.: Phys. Rev. Lett. 101, 077005 (2008)

10. Tinkham, M.: Introduction to Superconductivity. McGraw-Hill, New York (1975)

11. Dubroka, A., et al.: Phys. Rev. Lett. 101, 097011 (2008)

12. Shan, L., et al.: Europhys. Lett. 83, 57004 (2008)

13. Zhu, X., et al.: Supercond. Sci. Technol. 21, 105001 (2008)

14. Jia, Y., et al.: Appl. Phys. Lett. 93, 032503 (2008)

15. Angst, M., et al.: Phys. Rev. Lett. 88, 167004 (2002)

16. Lyard, L., et al.: Phys. Rev. Lett. 92, 057001 (2004)

17. Gurevich, A.: Phys. Rev. B 67, 184515 (2003)

18. Angst, M., Puzniak, R.: In: Martines, B.P. (ed.) Focus on Superconductivity, vol. 1, pp. 1-49. Nova Science, New York (2004). arXiv:cond-mat/0305048

19. Kogan, V.G.: Phys. Rev. B 24, 1572 (1981)

20. Kogan, V.G., Fang, M.M., Mitra, S.: Phys. Rev. B 38, 11958 (1988)

21. Zhigadlo, N.D., et al.: J. Phys.: Condens. Matter. 20, 342202 (2008) 
22. Karpinski, J., et al.: Supercond. Sci. Technol. 16, 221 (2003)

23. Karpinski, J., et al.: Physica C 385, 42 (2003)

24. Kohout, S., Roos, J., Keller, H.: Rev. Sci. Instr. 78, 013903 (2007)

25. Willemin, M., et al.: Phys. Rev. B 58, R5940 (1998)

26. Pugnat, P., Fillon, G., Noel, H., Barbara, B.: Europhys. Lett. 35, 49 (1996)

27. Kawamata, S., Inoue, K., Ochida, K., Sasaki, T.: Physica B 246, 437 (1998)

28. Hunte, F., et al.: Nature 453, 903 (2008)

29. Werthamer, N.R., Helfand, E., Hohenberg, P.C.: Phys. Rev. 147, 295 (1966)
30. Luetkens, H., et al.: Phys. Rev. Lett. 101, 097009 (2008)

31. Khasanov, R., et al.: Phys. Rev. B 78, 092506 (2008)

32. Rammer, J.: Europhys. Lett. 5, 77 (1988)

33. Matano, K., et al.: Europhys. Lett. 83, 57001 (2008)

34. Posazhennikova, A.I., Dahm, T., Maki, K.: Europhys. Lett. 60, 134 (2002)

35. Khasanov, R., et al.: Phys. Rev. Lett. 99, 237601 (2007) and Refs. therein 